\title{
Influencia de indicadores de resiliencia post COVID-19 en el diagnóstico urbano sostenible
}

\author{
Carolina Morales Robalino \\ lmoralesr@ulvr.edu.ec
}

Eugenia Dueñas Barberán mduenasb@ulvr.edu.ec

Jacqueline Luna Cabrera

Info@1laqtastudio.com

Universidad Laica Vicente Rocafuerte de Guayaquil

Guayaquil - Ecuador

\section{RESUMEN}

Este trabajo presenta el estudio de indicadores urbanos de resiliencia en el contexto de la pandemia COVID-19. El estudio se concentra en aspectos tales como proximidad a servicios y espacios públicos en el sector "La Garzota" de la ciudad de Guayaquil en Ecuador. La inclusión de indicadores dentro de los diagnósticos urbanos permite la construcción de una línea base para el monitoreo de respuestas emergentes frente a situaciones inciertas como una pandemia. Esto supone el control sobre el flujo de los objetivos trazados en la toma de decisiones. En este sentido, el trabajo analiza datos usando una metodología cuantitativa para establecer un diagnóstico territorial capaz de potenciar el bienestar de las localidades por medio de respuestas que generen cohesión social, identidad y acceso equitativo. Como resultado, el trabajo logra mapear los datos analizados con propuestas de intervención a nivel de criterios con el fin de brindar soluciones a las necesidades humanas identificadas.

Palabras clave: indicadores; sostenibilidad; desarrollo; resiliencia; COVID 


\title{
Influence of post-COVID-19 resilience indicators on sustainable urban diagnosis
}

\begin{abstract}
This paper presents a study on urban resilience indicators in the context of the COVID19 pandemic. The study centers its efforts around aspects such as proximity to services and public spaces in "La Garzota" area in Guayaquil, Ecuador. The inclusion of indicators as part of the urban diagnosis processes allows the determination of a baseline for monitoring the emergency responses to very uncertain situations such as a pandemic. This implies the control over the objectives traced during the decision making phase. In that context, this work analyzes data using a quantitative methodology to establish on site diagnosis capable of strengthening local development through social cohesion, identity and equitable access. The result of this work is a mapping of the analyzed data to intervention proposals in order to provide solutions to the identified human necessities
\end{abstract}

Keywords: indicators; sustainability; development; resilience; COVID

Artículo recibido: 10 Agosto. 2021 Aceptado para publicación: 07. Setiembre. 2021

Correspondencia: lmoralesr@ulvr.edu.ec Conflictos de Interés: Ninguna que declarar 


\section{INTRODUCCIÓN}

Los indicadores de "resiliencia urbana" (Hábitat III, 2015), constituyen uno de los elementos de estudio más importantes dentro del contexto de la pandemia del COVID 2019, para asegurar un proceso eficiente de recuperación económica y social. Según Hamby, S., et. al (2017, p.1), el término resiliencia se asocia a un proceso de tres factores: el primero, inicia con la aparición de un suceso estresante, el segundo, con la identificación de un espacio con funcionamiento saludable luego del suceso, y por último con la dotación de un mecanismo de acceso para la recuperación de las personas afectadas.

En este sentido, la inclusión de indicadores dentro de los diagnósticos urbanos sostenibles, da paso a la construcción de una línea base para el monitoreo de respuestas emergentes frente a situaciones inciertas; a saber, Tumini, I. (2016) refleja en su estudio que "entre la sostenibilidad y la resiliencia existen solapes y similitudes que pueden favorablemente aprovecharse como sinergias para el desarrollo de las ciudades" (p.15). De esta manera, se obtiene un control sobre el flujo de las acciones que se llevan cabo para mejorar las condiciones de habitabilidad.

Ahora bien, la restricción de movilidad provocada por la pandemia (Decreto Ejecutivo N. ${ }^{\circ} 1019$ de 2020, Ecuador), dejó en evidencia algunos problemas originados por la escasa planificación urbana con respecto a equipamientos; esto, dio paso a que los habitantes de varios sectores generen largos recorridos para tener acceso a servicios terciarios de uso cotidiano y a espacios verdes que les brinden un contacto con "espacios medioambientalmente saludables" (Merayo et.al, 2016, p. 5).

Por tal motivo, el argumento de este artículo se concentra en la aplicación de indicadores tales como la proximidad a los servicios y espacios públicos (Rueda, S. et. al, 2010; The Rockefeller Foundation \& ARUP, 2015; Huang, C. et.al., 2017), con el objetivo, de analizar datos cuantitativos que brinden un diagnóstico territorial capaz de potenciar el bienestar de las localidades por medio de la cohesión social, identidad y acceso equitativo. Bajo este escenario, De Sena, A. (2012) refleja que la identificación de estos indicadores es subjetiva al contexto y requiere de un sustento teórico que lo avale; es decir, que las dinámicas de interacción de la población son diferentes de acuerdo a muchos factores como la región, el entorno económico, social y ambiental, la caracterización del usuario 
y sus actividades. Por esta razón, se ha hecho énfasis en el análisis a escala barrial de un quintil intermedio, para determinar los datos de estas dos variables.

El sector La Garzota, se encuentra ubicado al norte de la ciudad de Guayaquil en Ecuador, con una extensión territorial de 120 hectáreas y una población estimada de 7.208 habitantes, de la cual el 57\% es económicamente activa. (Barros, C., 2013 \& INEC, 2010). Desde su creación en la década de los 80, se configura como un sector residencial cuyos espacios comerciales representan un apoyo en equipamientos para las zonas aledañas; por tal motivo, se identifica que la unidad tipológica habitacional corresponde a viviendas unifamiliares de 1 a 2 pisos y multifamiliares de 2 a 4 pisos dispuestas en una trama regular con vías abiertas de acceso principal y secundario.

A nivel climático, la zona posee una temperatura promedio en temporada calurosa de $24^{\circ} \mathrm{C}$ y en temporada fresca de $29^{\circ} \mathrm{C}$, con una humedad que varía considerablemente entre el $60 \%$ y $47 \%$, con diferentes promedios en la velocidad del viento que va desde el 9,9 $\mathrm{km} / \mathrm{h}$ más calmado hasta el $15,4 \mathrm{~km} / \mathrm{h}$ lo más ventoso

\section{Resiliencia en el contexto de la pandemia}

Desde este punto de vista Canosa Acosta, V. E., \& Gutiérrez Rodríguez, S. O. (2020) mencionan las experiencias en las emociones y el comportamiento de los seres humanos en sus etapas de crecimiento. Optar por una psicología positiva para tener calidad de vida se refleja en las manifestaciones sentimentales como producto de situaciones vividas en el tiempo.

Lo que antecede y lo que precede en el entorno familiar, social, económico y cultural estimulan esa capacidad para soportar adversidades frente a sucesos traumáticos y en su lucha se fortalece para conseguir felicidad y bienestar que se reflejan en la salud física y mental, con una resistencia psicológica llena de metas y acciones positivas para afrontar con el carácter suficiente y aprender de las fatalidades.

Se reconoce a la resiliencia de la ciudad como "la capacidad de una ciudad o región para resistir, absorber, adaptarse y recuperarse de choques agudos y tensiones crónicas para mantener en funcionamiento los servicios críticos, y monitorear y aprender de los procesos en curso a través de la ciudad y los colaboración regional, para aumentar las habilidades de adaptación y fortalecer la preparación anticipando y respondiendo adecuadamente a los desafíos futuros" (Smart Mature Resilience, 2016, como se citó en Marana, P. et.al., 2018, p.1) 


\section{Indicadores de resiliencia en las ciudades}

La resiliencia de las ciudades puede influir positiva o negativamente, de acuerdo a los procesos de densificación; es decir, mientras más gente y activos se concentran en las ciudades, hay una gama cada vez más compleja de los choques y tensiones, en función de los ámbitos naturales tales como efecto de pandemias y cambio climático, tecnológicos, crisis socio-económicas y político-culturales. (Habitat III, 2015).

De acuerdo a diversos autores, entre los que se sugieren a Ebru, A., (2017) y The Rockefeller Foundation \& ARUP, (2015), la información de estos indicadores debe llevarse sobre líneas de acción que permitan un enfoque centrado en los grupos vulnerables. Dentro de las variables de salud y bienestar, se incluye un análisis de acceso inclusivo a servicios básicos, partiendo de la cercanía que se tenga a los equipamientos; mientras que, con relación a la economía y sociedad, se menciona el apoyo a la comunidad local a través de la cohesión social e identidad local.

De la misma manera, para Infraestructura y ecosistemas, se menciona el riesgo integral junto al mapeo de exposición, la administración efectiva de los ecosistemas, la infraestructura flexible y redes de transporte diversas y eficientes. Finalmente, se alude al liderazgo y estrategias, a través de procesos consultivos de planificación y uso de suelo apropiado.

\section{Indicadores urbanos de sostenibilidad aplicados al diagnóstico de situación actual}

Huang, C. et.al., (2017) y Rueda, S. et. al. (2010), por su parte referencian entre las variables a la calidad del espacio público, donde analiza el acceso del viario peatonal, la percepción del verde urbano y el índice de habitabilidad en el espacio público. Luego, se realiza una transición a una variable de espacios verdes y movilidad para manejar la permeabilidad del suelo, la superficie verde por habitante, la proximidad a espacios verdes, la densidad y diversidad de árboles por cada tramo de calle, donde también se toma en cuenta el modo de desplazamiento de la población y la proximidad a redes.

Así mismo, referencia la complejidad urbana, con respecto al equilibrio entre actividad y residencia, las actividades de proximidad y el consumo de servicios básicos originados por el metabolismo urbano. Además, se hace alusión a la cohesión social para establecer la dotación de equipamientos y proximidad a los mismo. Es evidente entonces, tal como lo indica Serebrisky, T.et.al. (2020), que se requiere de una infraestructura sostenible próxima, para poder brindar espacios de interacción entre la comunidad con su entorno. 


\section{ESTRATEGIAS METODOLÓGICAS O MATERIALES Y MÉTODOS}

Para el diagnóstico de La Garzota se toma como base los indicadores de sostenibilidad que se encuentran vinculados a la resiliencia y que son compatibles con las necesidades obtenidas de la encuesta sobre las necesidades de espacios públicos en época de pandemia.

Posterior, mediante de una investigación descriptiva obtenida desde las fórmulas publicadas por Rueda, S. et. al. (2010), se obtiene como resultado los datos mapeados de los indicadores con el fin de brindar soluciones a las necesidades humanas identificadas a partir de dos indicadores generales: proximidad a servicios y acceso a espacios públicos.

\section{Indicadores de proximidad a servicios}

La proximidad en el espacio urbano es una expresión de la vecindad de la ciudad, afectando tanto a la generación de movilidad como a la cohesión social de los ciudadanos. (Tumini, I., 2016). En este contexto, se identifican los equipamientos de servicio existentes y su radio de influencia a 300m o 5 minutos para valorar la cobertura que tiene la población en función del acceso a servicios variados no repetitivos. Por ello, se emplea la fórmula de cobertura simultánea a 4 equipamientos, la cual debe cumplir un porcentaje mínimo de $75 \%$ y un óptimo del $100 \%$.

\section{Fórmula 1.}

\section{Proximidad a Equipamiento de servicios}

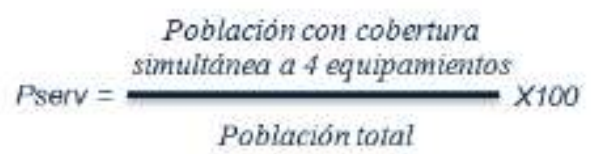

\section{Indicadores de Acceso a espacios públicos}

El análisis de los espacios públicos existentes se realiza con respecto a vías y espacios verdes, no se consideran las plazas puesto que todas ellas se encuentran bajo la categoría comercial y han sido analizadas en el apartado anterior. Para las vías se toma como base dos indicadores: el primero se relaciona con la accesibilidad del viario, cuyo criterio deseable es que su cobertura sea $>90 \%$ con aceras que contemplen una pendiente $<5 \%$ y una dimensión $>2.5 \mathrm{~m}$ mientras que el valor mínimo se configura en una acera $>0.9 \mathrm{~m}$ y los mismos valores de cobertura y pendiente que el óptimo. En este caso, se toma la fórmula detallada a continuación: 


\section{Fórmula 2.}

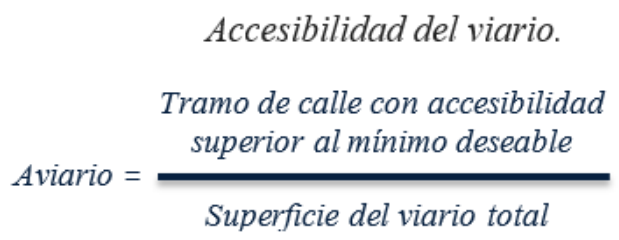

El segundo indicador de vías, se basa en la percepción del espacio verde urbano; es decir, el porcentaje de campo visual con vegetación en que el público tiene registro. Este análisis toma como volumen a la vegetación arbustiva mediana y alta, donde se plantea una cobertura mínima $>50 \%$ y deseable del $75 \%$, con la siguiente fórmula:

\section{Fórmula 3.}

Percepción del espacio verde urbano.

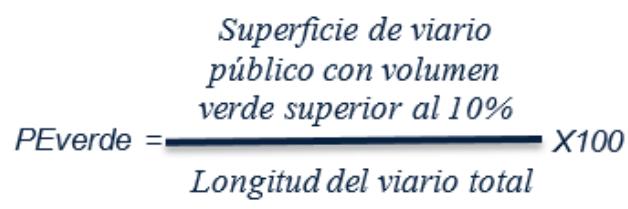

Por otro lado, para los espacios verdes, se analiza tomando como referencia los parques y jardines que se encuentran dentro del sector, sin la influencia de las áreas verdes aledañas de las otras urbanizaciones. Para ello, se analiza el índice verde urbano del sector, cual debe cumplir con dos requisitos mínimos, primero que obtenga un valor $>10 \mathrm{~m}^{2} /$ hab a pesar de que la Organización Mundial de la salud (OMS, 2016) recomiende como mínimo $9 \mathrm{~m}^{2} / \mathrm{hab}$. Y segundo, que su proximidad sea a $200 \mathrm{~m}$ de $\operatorname{los}$ usos residenciales. El análisis se lo realiza bajo la siguiente fórmula:

\section{Fórmula 4.}

\section{Superficie verde}

$$
\text { Sverde }=\frac{\text { Superficie verde }}{\text { Número de habitantes }}
$$

Bajo este orden, se menciona que el estudio hace énfasis en la presencia de zonas verdes, puesto que éstas se relacionan con mejores niveles de salud mental en la población, al actuar como reguladores de estrés, ansiedad y depresión, siempre que influyan en un radio 
menor a tres kilómetros dependiendo de su escala urbana y los servicios eco sistémicos que brinde (Merayo et.al, 2016)

\section{RESULTADOS Y DISCUSIÓN}

La Garzota posee una densidad poblacional bruta de 60.6 hab/ha y una estructura urbana consolidada, lo cual permite que las dinámicas poblacionales de movilidad y acceso, sean flexibles y adaptables. El diagnóstico muestra los siguientes resultados:

Del indicador de proximidad a los servicios, se obtiene que el $76.9 \%$ de la población posee una cobertura simultánea de 4 equipamientos; es decir, se identifica un incremento del 1.9\% sobre la ponderación mínima, que se atribuye a los usos de suelo comerciales y mixtos que se desarrollan a lo largo de las vías primarias que delimitan La Garzota y que a su vez comparten su radio de influencia sobre las ciudadelas aledañas a este sector.

A pesar de que en el estudio se evidencia un $23.1 \%$ faltante, no significa que la zona carezca de cobertura, sino que las áreas residenciales tienen acceso a 2 o 3 servicios dependiendo de su ubicación; esto se visualiza principalmente en las zonas céntricas, tal como lo indica la imagen 1.

\section{Imagen 1.}

\section{Proximidad a Equipamientos en La Garzota.}

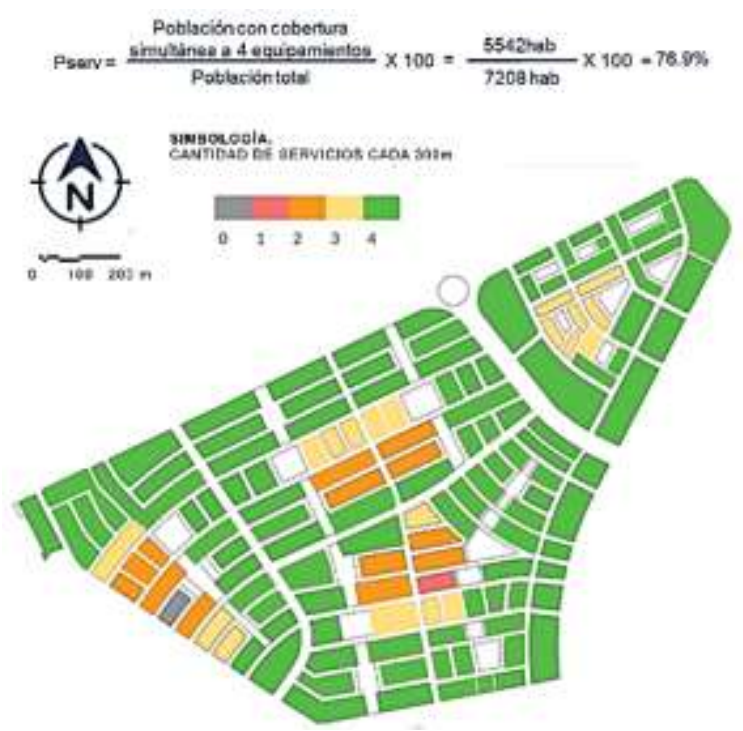

Con respecto a la accesibilidad del viario, se obtiene que el sector posee $90.3 \%$ de aceras con dimensiones mayores a $0.90 \mathrm{~m}$. Esto, se evidencia principalmente en vías primarias y secundarias, sin embargo, es preciso reconocer que la morfología de las aceras del viario, responde a una franja de servicios ocupada por jardinería, estacionamientos o mobiliario para infraestructura de redes que dificulta el recorrido peatonal. 
En concordancia al resultado obtenido, se estima que aproximadamente el $11 \%$ de este valor, cumple con las dimensiones deseables para la franja de circulación sin barreras arquitectónicas; no obstante, su configuración como espacio público, requiere de adecuaciones para que las personas desarrollen un sentido de apropiación y obtengan ambientes saludables.

Por otro lado, se manifiesta que el $9.7 \%$ faltante, no poseen las dimensiones necesarias de accesibilidad (Ver imagen 2), lo cual conlleva a que los peatones hagan uso de la vía vehicular para movilizarse y mantener el distanciamiento físico.

\section{Imagen 2.}

\section{Accesibilidad del viario}

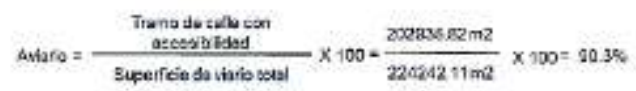

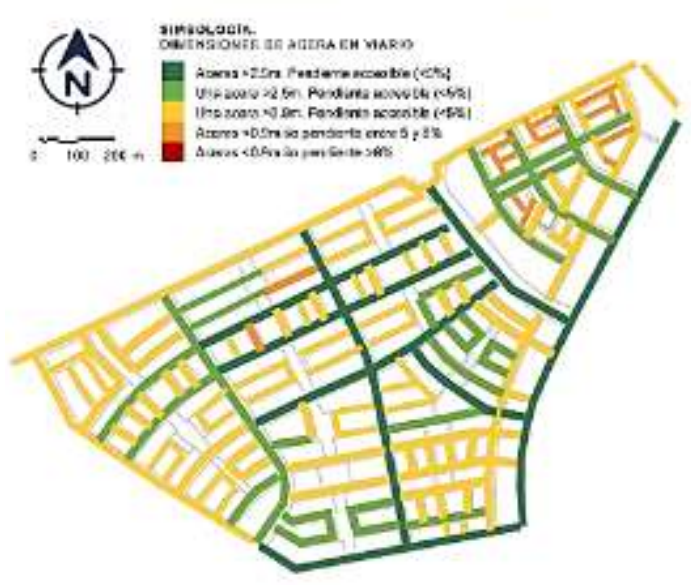

En el diagnóstico de la percepción verde urbano, se identifica que el 1.5\% de vías, cumple con las condiciones óptimas de brindar a los transeúntes, un registro visual con vegetación mediana y alta dentro del espacio público; es decir, que este indicador presenta un déficit de $48.5 \%$ para llegar al valor mínimo. Este resultado se obtiene por la configuración de las aceras, ya que su tipología plantea vegetación baja junto a las zonas residenciales ubicadas en vías secundarias.

En la imagen 3, se evidencia que a pesar de que las vías delimitantes, cumplen una función de vialidad principal, éstas no logran plasmar el mínimo requerido; esto se debe a que las vías poseen dimensiones mayores, pero no contemplan dentro de su zona de servicios, la dotación de vegetación media-alta que permita brindar a los transeúntes un espacio de descanso y confort. En el caso de las vías señaladas en rojo, no se registra ningún tipo de 
vegetación, debido a que el ancho de las vías solo contempla una zona de circulación peatonal con una franja de servicios destinada a la colocación de postes eléctricos.

\section{Imagen 3.}

\section{Percepción del verde urbano}

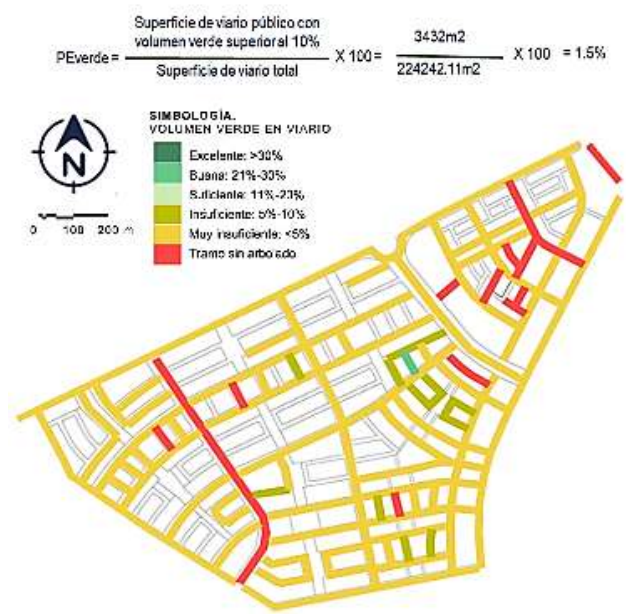

Finalmente, el análisis de la superficie verde, en la imagen 4, indica que La Garzota posee $14.77 \mathrm{~m} 2$ por habitante, lo cual representa un $39 \%$ superior al valor recomendado por la OMS y un $32 \%$ superior al valor mínimo del indicador. Este resultado se refleja por la morfología urbana de la ciudadela, la cual dispone de ejes centrales verdes entre las zonas residenciales que facilitan el acceso de los habitantes a zonas de recreación sin realizar largos recorridos.

La configuración de esta zona se encuentra estratégicamente diseñada, de manera que los habitantes del sector tienen acceso peatonal en menos de 5 minutos; sin embargo, el estudio refleja que aproximadamente un $40 \%$ de estos parques necesita de intervenciones que dinamicen las actividades de recreación y que a su vez generen cohesión social entre sus moradores. Para concluir, es preciso exaltar, que estas áreas verdes poseen vegetación alta que regula la sensación térmica del clima húmedo guayaquileño y que la eliminación de defensas permite su acceso de manera independiente y libre. 


\section{Imagen 4.}

\section{Superficie verde}

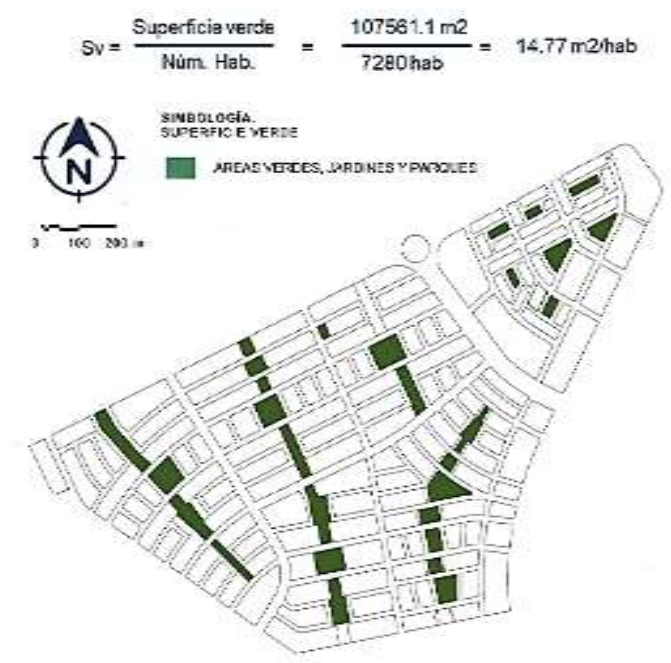

\section{CONCLUSIÓN O CONSIDERACIONES FINALES}

Ante la llegada del COVID-19, el mundo paralizó sus actividades por efecto de la cuarentena; durante estos meses, familias enteras tuvieron que buscar la manera de abastecerse y cubrir necesidades básicas, de salud y financieras. Justamente, esta situación permitió conocer las nuevas dinámicas de interacción de la población, especialmente por la necesidad de acceso a equipamientos y servicios.

Luego del aislamiento, la ciudad empezó a reactivarse con ciertas condicionantes fuera de lo habitual, tales como el distanciamiento físico y la movilidad controlada; éstas acciones han permitido evidenciar que, con relación al indicador de acceso a los servicios, se requiere de propuestas urbanas que permitan la planificación de actividades terciarias con menor recorrido. El diagnóstico de la Garzota manifiesta como la dotación de equipamientos permite cubrir las necesidades en radios de $300 \mathrm{~m}$, sin embargo, época de pandemia, es pertinente disminuir la influencia entre $100 \mathrm{~m}$ y $200 \mathrm{~m}$ de acceso peatonal.

La permeabilidad morfológica dentro de los contextos urbanos permite que el acceso a los servicios se dinamice, estableciendo una relación entre las necesidades humanas y la resiliencia urbana. Por ello, la proximidad es uno de los indicadores compatibles entre la sostenibilidad y resiliencia que se requieren dentro de los diagnósticos urbanos para mejorar las condiciones de habitabilidad.

Dinamizar las actividades urbanas en función de los factores ambientales que brinda el espacio público a nivel de diseño urbano, es otro de los indicadores de resiliencia que 
influyen positivamente dentro del diagnóstico local. La accesibilidad del viario, la percepción del verde urbano y el índice de áreas recreativas verdes, permiten contemplar los beneficios de los servicios ecosistémicos, especialmente en épocas de pandemia, puesto que se requiere de espacios saludables que brinden las condiciones necesarias de confort tales como oxigenación del espacio, reducción de las islas de calor, atracción de avifauna, la mejora de la imagen urbana, y la apropiación del espacio público con el distanciamiento físico requerido sin llegar a la privatización de las instancias.

En este sentido, la variable de la percepción de seguridad del barrio por parte de los ciudadanos en aceras y parques, podría mejorar a través de la dotación de árboles de copa ancha para el confort térmico e implementar elementos de descanso, de tal forma que el espacio público y el recorrido ofrezcan al peatón un programa más rico de actividades posibles para una estancia en las aceras y mayores recorridos peatonales dentro del sector. Para finalizar, se evidencia que realizar un diagnóstico urbano con indicadores relacionados a la resiliencia en pro de un urbanismo sostenible permite evidenciar problemas que muchas veces no han sido tomados en cuenta pero que se hacen más evidentes cuando ocurren circunstancias de mucha inestabilidad emocional en la ciudad. La resiliencia es la clave para transformar la ciudad luego de haber atravesado una pandemia y la mejor manera de empezar es identificando los puntos menos favorables dentro de los indicadores de sostenibilidad urbana.

\section{AGRADECIMIENTOS}

Este artículo reconoce la participación de Andrea Rosero, Alexander Burgos y Valeria Alarcón. Alumnos de la Universidad Laica Vicente Rocafuerte de Guayaquil en la materia de Diseño Urbano Ambiental, por su destacada participación en el mapeo territorial de los indicadores tratados.

\section{LISTA DE REFERENCIAS}

Barros, C. (20 de junio de 2013). La Garzota, 30 años de cambio. El Universo. https://www.eluniverso.com/noticias/2013/06/20/nota/1046291/garzota-30-anoscambios?amp

Canosa Acosta, V. E., \& Gutierrez Rodriguez, S. O. (2020). Resiliencia durante la cuarentena por COVID-19: El papel de las emociones y actividades saludables. [Tesis de grado, Universidad de La Laguna] 
De Sena, A. (2012) "¿Qué es un indicador?. Algunos elementos conceptuales en torno a la noción de indicador y su elaboración, pp. 161-189. Reflexiones y prácticas de la investigación social". ISBN 978-987-652- 117-8. Ediciones Cooperativas. Buenos Aires.

Decreto Ejecutivo N. 1019 de 2020. [Presidencia de la República del Ecuador] Por la cual se establece como zona especial de seguridad a toda la provincia del Guayas, a fin de mitigar los riesgos, precautelar la salud, proteger a la población, evitar el contagio del virus COVID-19. 22 de marzo de 2020. https://minka.presidencia.gob.ec/portal/usuarios_externos.jsf

Ebru, A. (2017). Cómo desarrollar ciudades más resilientes. Un Manual para líderes de los gobiernos locales. Ginebra, Marzo: UNISDR.

Hábitat III. (2015). Resiliencia Urbana. [Archivo PDF]. Conferencia de las Naciones Unidas sobre la vivienda y el desarrollo urbano sostenible

Hamby, S., Grych, J., \& Banyard, V. (2017). Resilience portfolios and poly-strengths: Identifying protective factors associated with thriving after adversity. Psychology of violence, 8(2), 172.

Huang, C., Busch, C., He, D., \& Harvey, H. (2017). green guidelines-CDBC's green and smart urban development guidelines.

INEC. (2010). Instituto Nacional de estadísticas y Censos. Obtenido de https://www.ecuadorencifras.gob.ec/institucional/home/

Marana, P., Labaka, L., \& Sarriegi, J. M. (2018). A framework for public-private-people partnerships in the city resilience-building process. Safety science, 110, 39-50.

Merayo Rodríguez, J., Serrano Fuentes, N., \& Marqués Sánchez, P. (2016). Influencia de los espacios verdes urbanos en la salud mental. Metas de Enfermería, 19(9), 2026.

Organización Mundial de la Salud (2016). Urban Green Space and Health: Intervention Impacts and effectiveness. https://www.euro.who.int/_data/assets/pdf_file/0007/338074/full-report-forarchiving.pdf?ua=1

Rueda, S, et. al. (2010). Plan de indicadores de sostenibilidad urbana de VitoriaGasteiz. Agencia de Ecología Urbana de Barcelona, Ayuntamiento Vitoria Gasteiz. 
Serebrisky, T., Brichetti, J. P., Blackman, A., \& Moreira, M. M. (2020). Infraestructura sostenible y digital para impulsar la recuperación económica post COVID-19 de América Latina y el Caribe: un camino hacia más empleo, integración y crecimiento. Banco Interamericano de Desarrollo.

The Rockefeller Foundation \& ARUP. (2015). City resilience Framework: Understanding measuring city resilience. https://www.arup.com/perspectives/publications/research/section/city -resilience-index

Tumini, I. (2016). Acercamiento teórico para la integración de los conceptos de Resiliencia en los indicadores de Sostenibilidad Urbana. Revista de Urbanismo, (34), 4-19. doi:10.5354/0717-5051.2016.40056 